\title{
Dynamics and chronology of Mycoplasma hyopneumoniae strain 232 infection in experimentally inoculated swine
}

\author{
Henrique M. S. Almeida ${ }^{1}$, Marina L. Mechler-Dreibi ${ }^{1}$, Karina Sonálio ${ }^{1}$, Marcela M. Ferreira ${ }^{1}$, Paulo E. B. Martinelli'
} Igor R. H. Gatto ${ }^{1}$, Dominiek Maes², Hélio J. Montassier ${ }^{1}$ and Luís G. Oliveira ${ }^{1 *}$ (D)

\begin{abstract}
Direct detection of Mycoplasma hyopneumoniae through molecular tools is a growing trend for early diagnosis, highlighting the importance of knowing $M$. hyopneumoniae dynamics in the respiratory tract upon infection. This study focused on monitoring the infection level and its effects in different anatomic sites of the respiratory tract of experimentally infected swine in four time-points post-infection. To this end, 24 pigs were allocated to either noninoculated group $(n=8)$ or inoculated group $(n=16)$. On day 0 post-infection (dpi), animals of the inoculated group were intratracheally inoculated with $M$. hyopneumoniae. Nasal swabs were collected weekly for qPCR detection of bacterial shedding. At 14, 28, 42, and $56 \mathrm{dpi}$, four animals from the inoculated group and two from the control group were necropsied. Bronchoalveolar lavage fluid (BALF) and samples from three different anatomical tracheal sections (cranial - CT, medium - MT, lower - LT) were collected for QPCR and histopathology. Bacterial loads (qPCR) in tracheal samples were: $4.47 \times 10^{2}$ copies / $\mu \mathrm{L}(\mathrm{CT}), 1.5 \times 10^{4}$ - copies / $\mu \mathrm{L}(\mathrm{MT})$ and $1.4 \times 10^{4}$ copies $/ \mu \mathrm{L}$ (LT samples). M. hyopneumoniae quantification in BALF showed the highest load at $28 \mathrm{dpi}\left(2.0 \times 10^{6}\right.$ copies $\left./ \mu \mathrm{L}\right)$. Microscopic lesions in LT samples presented the highest scores at $56 \mathrm{dpi}$ and were significantly correlated with the pathogen load on $14 \mathrm{dpi}(0.93)$ and $28 \mathrm{dpi}(0.75)$. The greatest bacterial load of M. hyopneumoniae in CT samples and BALF was registered at $28 \mathrm{dpi}$, and it remained high in BALF and LT throughout the $56 \mathrm{dpi}$. The pathogen was able to persist during the whole experimental period, however higher estimated quantification values were registered in the lower parts of the respiratory tract, especially at $56 \mathrm{dpi}$. These findings are important for improving diagnostics, treatment, and control measures of M. hyopneumoniae infection in swine herds.
\end{abstract}

Keywords: Enzootic pneumonia, qPCR, Infectious diseases, Respiratory diseases, Diagnostics

\section{Background}

Mycoplasma hyopneumoniae is the primary causative agent of enzootic pneumonia (EP) [1], a chronic disease characterized by dry, non-productive cough, high morbidity, low mortality, and losses in daily weight gain [2, 3]. The bacterium enters the host's organism through

\footnotetext{
* Correspondence: luis.guilherme@unesp.br

${ }^{1}$ São Paulo State University (Unesp), School of Agricultural and Veterinarian Sciences, Via de Acesso Prof. Paulo Donato Castellane s/n, Jaboticabal, SP 14884-900, Brazil

Full list of author information is available at the end of the article
}

the upper respiratory tract and adheres to the ciliated epithelium of the trachea, bronchus, and bronchi [4], disrupting the mucociliary defensive system and increasing infection susceptibility for secondary respiratory pathogens, which commonly results in co-infections, like the porcine respiratory disease complex (PRDC) [5].

$M$. hyopneumoniae is known for having a tropism for lower parts of the swine respiratory tract [6-9]. The population of this bacteria was shown to be 100 times higher in these sites when compared to the upper respiratory tract [6]. By assessing the lungs of 
experimentally infected pigs, $M$. hyopneumoniae was detected in bronchus and bronchioles as early as 7 days post-infection (dpi). Simultaneously, its direct detection in the alveolar macrophage and interstitial macrophages was only seen in the later stages of infection [7, 9].

Still, M. hyopneumoniae is notorious for its specific membrane proteins that adhere to the tracheal epithelial cilia during infection [10], inducing tissue inflammation and cell damage in vitro [11]. Thus, collection of tracheal secretions, using tracheobronchial swabs (deep tracheal catheters) and laryngeal swabs, have been proven to be sensitive and reliable diagnostic samples to detect M. hyopneumoniae infection in live animals [12, 13]. Consequently, it is very likely that the trachea is also an infection and multiplication site for M. hyopneumoniae [14]. However, as far as we know, there are no studies that looked into the distribution of the pathogen in the trachea throughout infection.

The knowledge about the dynamics and chronology of $M$. hyopneumoniae infection is essential for decisionmaking in the sampling methodology [15]. However, currently, there is little information regarding the bacterial load in different parts of the respiratory tract during infection. Considering the aforementioned fact, this study focused on reporting the infection dynamics upon experimental $M$. hyopneumoniae challenge and the associated effects in different respiratory tract sections of swine during a period of $56 \mathrm{dpi}$.

\section{Results}

\section{Presence of clinical signs in inoculated piglets}

The laryngeal swab and serum samples collected before inoculation were negative for M. hyopneumoniae DNA (qPCR) and specific M. hyopneumoniae antibodies (ELIS A), respectively. While in the mock-inoculated animals, clinical signs compatible with EP were not detected, all inoculated animals presented clinical signs consistent with EP. The dry, non-productive cough was first noted at $10 \mathrm{dpi}$ and thereafter observed in all inoculated animals. At the end of the experiment (56 dpi), at least one animal was still coughing. Beyond that, the animals did not show any clinical signs associated with other diseases.

\section{Detection of $M$. hyopneumoniae DNA in biological samples qPCR parameters}

All qPCR parameters followed the previously published recommendations described in the Minimum Information for Quantitative Real-Time qPCR Experiments (MIQE) [16]. The efficiency ranged from 92 to $98.3 \%, R^{2}$ ranged from 0.996 to 1.0, y-intercept ranged from 39 and 40.8 , and the slope varied from -3.36 to -3.5 .

\section{Nasal swabs samples}

Mock-inoculated animals remained negative during the whole study. The presence of M. hyopneumoniae DNA in nasal swab samples was detected from seven dpi (616) onwards, and all animals, except for pig 2.4, presented at least one positive result across the study. Most animals showed positive results at $14 \mathrm{dpi}$ and $21 \mathrm{dpi}$, $75 \%(12 / 16)$ and $91.6 \%$ (11/12), respectively. Bacterial shedding was shown to have an intermittent pattern, as reported in Table 1. The average Cq value obtained for each sampling day and the respective standard deviation are also shown in Table 1.

\section{Tracheal samples}

No positive results were obtained from the samples of mock-infected animals. The median of M. hyopneumoniae DNA quantification value in CT, MT, and LT samples at 14, 28, 42, and $56 \mathrm{dpi}$ are shown in Table 2. Despite the numeric difference, no significant differences were found between M. hyopneumoniae burden in CT, MT and LT and dpi.

\section{BALF samples}

All BALF samples from mock-inoculated animals were negative by qPCR during the whole study, while samples from inoculated animals were positive. Significant differences $(p<0.05)$ were only detected between the mean values at $14 \mathrm{dpi}$ and $28 \mathrm{dpi}$. These results are shown in Table 2.

\section{Gross lung lesion scoring (GLLS) and microscopic lesion scoring in tracheal samples}

Higher GLLS were seen at $14 \mathrm{dpi}$ and $28 \mathrm{dpi}$ than at 42 dpi and 56 dpi. Despite the numeric differences between means, no significant differences were detected using Tukey's test $(p<0.05)$. Scar tissue and gray fish-flesh-like areas were noted at $42 \mathrm{dpi}$ and $56 \mathrm{dpi}$. No gross lesions were observed in the lungs of the mock-inoculated group.

Results of cranial trachea lesion scores (CTLS), medium trachea microscopic lesion score (MTLS), lower trachea lesion score (LTLS), and GLLS are shown in Table 3. No significant differences were found in tracheal lesions scores between time points or anatomical regions when using the Tukey test or linear mixedeffects model test adjusted for Tukey $(p<0.05)$.

\section{M. hyopneumoniae detection in lung samples}

All lung samples from inoculated animals were positive for M. hyopneumoniae using qPCR. In contrast, lung tissue samples from mock-inoculated animals did not show any positive results in the qPCR test. 
Table $1 \mathrm{Cq}$ values obtained in qPCR of nasal swabs samples for detection of M. hyopneumoniae strain 232 in experimentally infected pigs throughout 56 dpi

\begin{tabular}{|c|c|c|c|c|c|c|c|c|c|c|}
\hline \multirow[t]{2}{*}{ Animal ID } & \multicolumn{10}{|l|}{ DPI } \\
\hline & -7 & 0 & 7 & 14 & 21 & 28 & 35 & 42 & 49 & 56 \\
\hline 1.1 & - & - & - & 35.9 & & & & & & \\
\hline 1.2 & - & - & 36.3 & 37.5 & & & & & & \\
\hline 1.3 & - & - & 37.5 & 24.0 & & & & & & \\
\hline 1.4 & - & - & - & 21.4 & & & & & & \\
\hline 2.1 & - & - & - & 37.3 & 36.3 & - & & & & \\
\hline 2.2 & - & - & - & 35.5 & 35.0 & - & & & & \\
\hline 2.3 & - & - & - & 32.8 & 35.6 & - & & & & \\
\hline 2.4 & - & - & - & - & - & - & & & & \\
\hline 3.1 & - & - & 38.0 & 36.4 & 36.1 & 36.3 & 38.6 & 38.0 & & \\
\hline 3.2 & - & - & - & - & 36.6 & - & - & 38.1 & & \\
\hline 3.3 & - & - & 37.5 & 37.1 & 36.4 & - & 37.8 & - & & \\
\hline 3.4 & - & - & 37.5 & - & 34.9 & 35.9 & 39.4 & 36.3 & & \\
\hline 4.1 & - & - & - & - & 36.7 & 37.0 & - & 34.8 & 36.3 & - \\
\hline 4.2 & - & - & - & 38.0 & 36.5 & - & 37.1 & 39.4 & 36.2 & 37.8 \\
\hline 4.3 & - & - & 37.3 & 36.1 & 37.2 & 37.2 & 37.0 & - & - & 37.0 \\
\hline 4.4 & - & - & - & 37.2 & 36.1 & 37.5 & 37.3 & 38.4 & 38.8 & - \\
\hline Cq Mean $( \pm S D)$ & & & $37.4( \pm 0.6)$ & $31.1( \pm 5.5)$ & $36.1( \pm 0.7)$ & $36.8( \pm 0.7)$ & $37.86( \pm 1.0)$ & $37.5( \pm 1.7)$ & $37.1( \pm 1.5)$ & $37.4( \pm 0.6)$ \\
\hline Total $^{\mathrm{a}}$ & $0 / 16$ & $0 / 16$ & $6 / 16$ & $12 / 16$ & $11 / 12$ & $5 / 12$ & $6 / 8$ & $6 / 8$ & $3 / 4$ & $2 / 4$ \\
\hline
\end{tabular}

${ }^{\mathrm{a}}$ Total expressed in positive / total sampled animals

\section{Correlations of $M$. hyopneumoniae load, GLLS, and tracheal sections lesion score}

M. hyopneumoniae estimated load in BALF samples was significantly correlated with GLLS at 14 (0.93), 42 (0.95), and 56 dpi (0.89). Regarding tracheal samples, M. hyopneumoniae estimated load in CT was only correlated with GLLS at $28 \mathrm{dpi}$ (0.86), while the load in MT correlated with GLLS at $28(0.78)$ and $56 \mathrm{dpi}$ (1.0) and with MTLS at $14 \mathrm{dpi}$ (0.89). Finally, the load in LT was correlated with LTLS at 14 dpi (0.93). All coefficient values obtained for significant correlations and the significance values at all four timepoints are shown in Table 4 .

\section{Discussion}

Understanding M. hyopneumoniae dynamics is essential for accurate diagnosis of infection, as well as for optimizing treatment and control measures in affected herds. Therefore, this study focused on reporting the infection dynamics upon experimental M. hyopneumoniae infection in specific sites (nasal swabs, CT, MT, LT, and bronchial tree) of the respiratory tract during a period of 56 dpi. To this end, M. hyopneumoniae DNA was quantified by qPCR in BALF and three anatomical sections of the trachea. In addition, gross Mycoplasma-like lung lesions and microscopic lesions of the trachea were scored.

Table 2 Median and the range of quantification of P102 fragment copies / $\mu \mathrm{L}$ of M. hyopneumoniae strain 232 in BALF, cranial, medium and lower trachea samples at four different dpi. Different superscript letters in the columns indicate significant differences at $p<0.05$ between dpi in Dunn's test

\begin{tabular}{lllll}
\hline Dpi & Sample & & & \\
\cline { 2 - 5 } & CT & MT & LT & BALF \\
\hline $\mathbf{1 4}$ & $1.0 \times 10^{2}\left(4.4 \times 10^{0}-1.23 \times 10^{4}\right)^{\mathrm{a}}$ & $1.3 \times 10^{4}\left(1.0 \times 10^{1}-1.9 \times 10^{4}\right)^{\mathrm{a}}$ & $3.8 \times 10^{4}\left(4.2 \times 10^{1}-7.58 \times 10^{4}\right)^{\mathrm{a}}$ & $5.7 \times 10^{4}\left(4.0 \times 10^{3}-1.2 \times 10^{5}\right)^{\mathrm{b}}$ \\
$\mathbf{2 8}$ & $4.6 \times 10^{3}\left(3.98 \times 10^{2}-1.53 \times 10^{6}\right)^{\mathrm{a}}$ & $3.2 \times 10^{2}\left(1.22 \times 10^{1}-1.05 \times 10^{3}\right)^{\mathrm{a}}$ & $1.0 \times 10^{4}\left(1.9 \times 10^{1}-1.15 \times 10^{5}\right)^{\mathrm{a}}$ & $1.2 \times 10^{6}\left(7.28 \times 10^{5}-2.0 \times 10^{6}\right)^{\mathrm{a}}$ \\
$\mathbf{4 2}$ & $1.6 \times 10^{1}\left(1.0 \times 10^{1}-5.12 \times 10^{2}\right)^{\mathrm{a}}$ & $8.1 \times 10^{3}\left(1.47 \times 10^{1}-1.44 \times 10^{5}\right)^{\mathrm{a}}$ & $5.0 \times 10^{3}\left(1.0 \times 10^{2}-2.0 \times 10^{4}\right)^{\mathrm{a}}$ & $1.9 \times 10^{5}\left(2.2 \times 10^{3}-7.6 \times 10^{5}\right)^{\mathrm{ab}}$ \\
$\mathbf{5 6}$ & $4.1 \times 10^{3}\left(2.0 \times 10^{2}-1.37 \times 10^{4}\right)^{\mathrm{a}}$ & $2.0 \times 10^{5}\left(8.1 \times 10^{1}-6.0 \times 10^{5}\right)^{\mathrm{a}}$ & $2.0 \times 10^{5}\left(8.7 \times 10^{3}-7.3 \times 10^{5}\right)^{\mathrm{a}}$ & $4.5 \times 10^{5}\left(1.6 \times 10^{4}-9.6 \times 10^{5}\right)^{\mathrm{ab}}$ \\
Median & $4.47 \times 10^{2}$ & $1.47 \times 10^{3}$ & $1.44 \times 10^{4}$ & $4.98 \times 10^{5}$ \\
\hline
\end{tabular}


Table 3 Mean gross lung lesion score (GLLS) and microscopic lesion scoring (standard deviation) in samples from three different anatomical sites of the trachea at different days post-infection (DPI): Cranial trachea (CT), Medium trachea (MT), and Lower trachea (LT) of experimentally inoculated animals with M. hyopneumoniae strain 232. Lungs were scored using the Christensen et al. (1999) [17] methodology. No significant differences $(P<0.05)$ were found using Tukey test or linear mixed-effects model test adjusted for Tukey

\begin{tabular}{|c|c|c|c|c|c|c|c|c|}
\hline \multirow[t]{2}{*}{$D P I$} & \multicolumn{4}{|l|}{ Control } & \multicolumn{4}{|l|}{ Inoculated } \\
\hline & GLLS (\%) & $\mathrm{CT}$ & MT & LT & GLLS (\%) & $\mathrm{CT}$ & MT & LT \\
\hline 14 & $0.0(0.0)$ & $0.5( \pm 0.5)$ & $0(0.0)$ & $0(0.0)$ & $13.43(2.21)$ & $1.0(0.82)$ & $1.75(1.7)$ & $2.75(1.5)$ \\
\hline 28 & $0.0(0.0)$ & $0( \pm 0.0)$ & $0(0.0)$ & $0(0.0)$ & $13.22(7.07)$ & $2.25(1.71)$ & $1.75(0.38)$ & $2.75(0.96)$ \\
\hline 42 & $0.0(0.0)$ & $0( \pm 0.0)$ & $0(0.0)$ & $0(0.0)$ & $9.61(7.0)$ & $0.75(0.5)$ & $1.75(0.5)$ & $2.25(1.5)$ \\
\hline 56 & $0.0(0.0)$ & $0.5( \pm 0.5)$ & $0.5(0.5)$ & $0.5(0.5)$ & 8.75 (5.72) & $1.5(1.0)$ & $3.0(0.81)$ & $3.0(0.81)$ \\
\hline
\end{tabular}

Throughout the experimental period ( 0 to $56 \mathrm{dpi}$ ), the highest $M$. hyopneumoniae loads in tracheal sections (assessed by qPCR testing) were detected at $28 \mathrm{dpi}$ $\left(4.6 \times 10^{3} \operatorname{copies} \mu \mathrm{L}\right)$ for $\mathrm{CT}$, and at $56 \mathrm{dpi}$ for MT $(2.0 \times$ $10^{5}$ copies $\left.\mu \mathrm{L}\right)$ and LT $\left(1.9 \times 10^{5}\right.$ copies $\left./ \mu \mathrm{L}\right)$ samples. Even though no statistical significance was found between $M$. hyopneumoniae quantification values in the tracheal sections, a numeric increase in M. hyopneumoniae load was observed in MT samples over time. Likewise, the load remained consistently high in LT samples throughout the whole studied period.

This pathogen is known for having tropism for lower parts of the swine's respiratory tract, being up to 100 times more common in such sites [6]. Previous studies have also shown that $M$. hyopneumoniae is more accurately detected in samples collected from the lower respiratory tract; consequently, lower $\mathrm{Cq}$ values were obtained from deep tracheal swabs than from nasal and tonsil swab samples in a previous report [15]. A more recent study showed a significantly higher sensitivity for deep tracheal catheter (sensitivity range: 0.71-0.94) samples when compared to laryngeal swabs $(0.27-0.57)$, mainly during late stages of infection (113 dpi) [12].

The abovementioned findings are in accordance with this study's results, in which higher M. hyopneumoniae loads were reported in the medium and lower tracheal sections. This fact is mostly related to the better diagnostic sensitivity found in samples obtained from such anatomical regions, like the deep tracheal catheters $[12,13]$.

The first step of $M$. hyopneumoniae infection is adhesion to ciliated cells of trachea, bronchi, and bronchioles mucosal epithelial lining, leading to inflammatory cells' infiltration and their accumulation around airways and blood vessels [18]. The lesions described above were also noted in tracheal samples from inoculated animals. Even though no significant differences were found, the scores seemed to increase over time, since the animals at 56 dpi presented the highest scores.

An in vitro inoculation of M. hyopneumoniae in cultured porcine tracheal cells showed progressive colonization and damage of the mucosal epithelial lining, noted from the 5th dpi onwards and with increasing severity over time [19], corroborating our microscopic lesion score results. In addition, a significant correlation between M. hyopneumoniae burden inoculated in swine tracheal cell culture with cilia loss in the same tissue [20], pathological alterations, and apoptosis [21], was reported, showing that the severity of the lesion may be affected by the pathogen infection load on-site.

In this research, LT samples showed numerically higher microscopic lesion scores when compared with MT and, especially, with CT. Interestingly, significant correlations were found at $14 \mathrm{dpi}$ between $M$.

Table 4 Significant correlations coefficients ( $r$ ) found between M. hyopneumoniae (MHP) quantification in trachea samples (Cranial trachea - CT; Medium trachea- MT and Lower trachea- LT) and bronchoalveolar lavage fluid (BALF) on the one hand and gross lung lesion score (GLLS) and microscopic lesion score in cranial (CTLS), medium (MTLS) and lower trachea (LTLS) on the other hand at different days post-infection (dpi)

\begin{tabular}{|c|c|c|c|c|c|c|c|c|c|c|c|c|c|c|c|c|}
\hline \multirow{2}{*}{$\begin{array}{l}\text { MHP } \\
\text { quantification }\end{array}$} & \multicolumn{4}{|c|}{$14 \mathrm{dpi}$} & \multicolumn{4}{|c|}{$28 \mathrm{dpi}$} & \multicolumn{4}{|c|}{$42 \mathrm{dpi}$} & \multicolumn{4}{|c|}{$56 \mathrm{dpi}$} \\
\hline & GLLS & CTLS & MTLS & LTLS & GLLS & CTLS & MTLS & LTLS & GLLS & CTLS & MTLS & LTLS & GLLS & CTLS & MTLS & LTLS \\
\hline $\mathrm{CT}$ & - & - & - & - & $0.86^{*}$ & - & - & - & - & - & - & - & - & - & - & - \\
\hline MT & - & - & $0.89^{*}$ & - & $0.78^{*}$ & - & - & - & - & - & - & - & $1.0^{* * *}$ & - & - & - \\
\hline LT & - & - & - & $0.93^{* *}$ & - & - & - & $0.75^{*}$ & - & - & - & - & - & - & - & - \\
\hline BALF & $0.93^{* *}$ & - & - & - & - & - & - & - & $0.95^{* *}$ & - & - & - & $0.89^{*}$ & - & - & - \\
\hline
\end{tabular}

${ }^{*} p<0.05 * * 0<0.01{ }^{* * *} p<0.001$ 
hyopneumoniae load in MT (0.89) and at 14 and $28 \mathrm{dpi}$ in LT (0.93 and 0.75, respectively) samples. Besides, as reported previously, the high load of M. hyopneumoniae found in these samples seems to play a role in the severity of microscopic lesion development in the first weeks of infection [11, 19].

Regarding the burden in BALF, the highest M. hyopneumoniae quantification value was reported at $28 \mathrm{dpi}$ $\left(1.2 \times 10^{6} \operatorname{copies} \mu \mathrm{L}\right)$. Since it did not significantly decrease under the present conditions, the pathogen possibly could have persisted for a longer period and therefore not cleared from the body after $56 \mathrm{dpi}$. In addition, a previous study showed that all experimentally infected animals were positive up to the $94 \mathrm{dpi}$, and approximately $60 \%$ had positive bronchial swabs results at 214 dpi [22].

Interestingly, the M. hyopneumoniae load in BALF samples was significantly correlated with GLLS at 14, 42, and $56 \mathrm{dpi}$, highlighting the importance of its burden in lesion severity. Likewise, Vranckx et al. [2] reported a similar correlation between the pathogen load in lung lesion samples and GLLS.

In this study, the mean M. hyopneumoniae load in CT and GLLS reached the highest values at $28 \mathrm{dpi}$ and were significantly correlated (0.86). Another study showed that $M$. hyopneumoniae load in nasal swab samples was significantly correlated to the load present in lung lesion homogenate of the same animal, showing that $M$. hyopneumoniae cells present in cranial regions of the respiratory tract are probably originated from lung lesions [6]. Therefore, it is possible to hypothesize that sampling the upper respiratory tract (i.e. nasal and laryngeal swabs) could result in the accurate detection of M. hyopneumoniae, considering the chronology of the disease. Nevertheless, further studies are necessary to achieve more accurate results regarding the association between diagnostic samples sensitivity and GLLS at the early stage of infection.

Additionally, on the $56 \mathrm{dpi}$, GLLS showed its lowest percentage $(8.75 \%)$ while the LT pathogen load was still high. This result agrees with previous findings, which confirmed that sampling lower respiratory tract sites would be more accurate for $M$. hyopneumoniae detection in later or chronic stages of the disease [12, 13, 23] when clinical signs are mild, and GLLS is regressing.

M. hyopneumoniae was first detected in nasal swabs at seven dpi and presented the highest number of positive animals at 21-28 dpi (92 and 81\%, respectively), allowing us to hypothesize that this was the time point when most animals were shedding the agent. Moreover, the detection of the pathogen in swab samples as early as five dpi and 28-30 dpi (peak) was already reported in other studies, as well as the intermittent detection pattern $[6,24,25]$.
Unfortunately, the Monte Carlo effect [26] is an inherent limitation of the qPCR technique commonly seen in samples with a low target DNA copy concentration. In such samples, quantification cannot be accurately performed since the Cq values obtained between duplicates vary significantly. Thus, it is likely that this effect prevented us from properly quantify $M$. hyopneumoniae shedding in nasal swab samples.

The present results originated from an experimental infection using intratracheal inoculation of one particular strain of M. hyopneumoniae (232). The experimental infection model used in this study was successful since all inoculated pigs were confirmed to be positive through qPCR and developed M. hyopneumoniae-like lesions in the lungs, as well as associated clinical signs.

It is worth mentioning that the experimental infection conditions were different from those on the field, mainly because of the infecting dose, the pathogen inoculation site, and housing conditions (such as dust, humidity, and air circulation within the barn). Consequently, the presented results should be extrapolated to field animals with caution, even though some basic principles such as the dynamics of the infection and the M. hyopneumoniae burden in tracheal sites may be valid under practical situations. It is also fair to acknowledge that the limited number of animals used in each group could be associated with the wide range of quantification results reported within groups.

Finally, even though qPCR is a sensitive technique for detecting pathogens in biological samples, it is essential to mention that it detects not only live M. hyopneumoniae cells but also dead ones. Consequently, the quantification results could be slightly overestimated.

\section{Conclusion}

The amount of M. hyopneumoniae genome fragments in the lower tracheal samples and BALF were high and remained relatively stable throughout $56 \mathrm{dpi}$. The CT samples' load was numerically high at $28 \mathrm{dpi}$ and associated with GLLS. Microscopic tracheal lesion scores seemed to increase with time and were more prominent in LT. To the best of our knowledge, this is the first study to experimentally assess the M. hyopneumoniae burden in different tracheal anatomical sections throughout $56 \mathrm{dpi}$, demonstrating the association of lung lesion and the pathogen dynamics in the respiratory tract.

\section{Methods}

Study design and experimental infection

A total of 24 male Large White pigs of 28-days of age were selected for this study. The animals originated from an M. hyopneumoniae, Actinobacillus pleuropneumoniae, and Porcine Reproductive and Respiratory 
Syndrome virus (PRRSv) free farm (since Brazil is a PRSS free country), being vaccinated only against swine influenza virus and porcine circovirus 2 (PCV2) with commercial vaccines. Moreover, the pigs remained without any noticeable clinical signs of infectious diseases until the inoculation. The animals were allocated in non-inoculated $(n=8)$ and inoculated $(n=16)$ groups. Each group was housed in isolated facilities (four animals per pen) with free access to water and fed twice a day, with an acclimatization period of 7 days before the inoculation. Laryngeal swabs and blood samples were collected on the arrival day (dpi - 7) and on the inoculation day (dpi0) to confirm the $M$. hyopneumoniae -free status by qPCR and ELISA (Mhyo atb test, IDEXX, USA), respectively.

At dpi 0 , control animals $(n=8)$ were mock-inoculated with $10 \mathrm{~mL}$ of sterilized Friis medium. The inoculated animals $(n=16)$ were given $10 \mathrm{~mL}$ of macerated lung inoculum (from experimentally infected cesarean-derived colostrum-deprived pigs; obtained from Iowa State University) containing $10^{6} \mathrm{CCU} / \mathrm{mL}$ of $M$. hyopneumoniae strain 232 (moderate virulence) diluted in sterilized Friis medium. Inoculation was performed with a laryngoscope's aid to introduce a sterile catheter in the trachea until the trachea bifurcation (approximately $20 \mathrm{~cm}$ deep) [18]. After the inoculation, the catheter was rinsed with $10 \mathrm{~mL}$ of sterilized physiological solution to ensure that the inoculum was completely administered. Then, the animals were clinically examined daily to detect possible clinical signs related to $M$. hyopneumoniae infection.

Every 14 days, at $14 \mathrm{dpi}, 28 \mathrm{dpi}, 42$, and $56 \mathrm{dpi}$, two control animals and four infected animals were randomly selected and euthanized according to the guidelines of the Brazilian Federal Veterinary Medicine council: $1 \mathrm{mgKg}$ bodyweight of Xylazine (Anasedan, Ceva) by intramuscular injection, followed by intracardiac injection of propofol (16 mgKg) (Propovan, Cristália - Brazil). After the euthanasia, a necroscopic evaluation was performed, along with the score of gross lung lesions and the collection of biological samples for further analysis.

\section{Collection of nasal swabs, bronchoalveolar lavage fluid (BALF), lung, and tracheal samples}

Nasal swabs were collected weekly using sterilized cotton swabs (Absorve, Brazil), which were inserted in both pig's nostrils, rotated three times clockwise, placed into DNAse and RNAse free microtubes (Axygen, USA) containing $500 \mu \mathrm{L}$ of sterilized Phosphate Buffered Saline (PBS) pH 7.4 (Sigma- Aldrich, USA), and kept at $-80^{\circ} \mathrm{C}$ until processing.

During necropsy, the respiratory tract from the larynx to the lungs was removed from the carcass and placed on a separate table. BALF samples were collected by injecting $15 \mathrm{~mL}$ of sterilized PBS (pH 7.4) (Sigma-Aldrich, USA) in the bronchial bifurcation with the aid of a sterilized glass pipette and an automatic pipettor. The lungs were gently massaged, and then the PBS was pulled back, placed into $1.5 \mathrm{~mL}$ DNAse and RNAse free cryovials (Corning, USA), and immediately stored at $80^{\circ} \mathrm{C}$ until use.

Samples of gross cranio-ventral consolidation lesions, considered characteristic of $M$. hyopneumoniae infection, were collected to confirm its involvement in the lung lesions seen at the necropsy. Fragments of approximately $1 \mathrm{~cm}^{3}$ were collected in the transition area of affected and healthy tissue using sterilized scalpel and forceps, instantly placed in DNAse and RNAse free cryovials (Corning, USA), and stored at $-80^{\circ} \mathrm{C}$ until use. Tissue samples of mock-inoculated animals were collected from all lung lobes since no gross lesions were seen during necropsy. For the inoculated animals with lesions in more than one lung region, samples were collected from all lesions and pooled into the same container.

Besides, tracheal samples from three different anatomic sites, namely, cranial trachea (CT) (from the larynx to the 12th tracheal ring), medium trachea (MT) (from 13th ring to the 24th tracheal ring), and lower trachea (LT) (from 25th tracheal ring until the bronchus bifurcation), were collected from each animal. Each tissue sample was divided into two; one was stored in individual DNAse and RNAse free microtubes (Axygen, USA) and immediately stored at $-80^{\circ} \mathrm{C}$ while the other was stored in $10 \%$ buffered formalin for $24 \mathrm{~h}$ and later transferred to $70 \%$ alcohol.

\section{DNA extraction from biological samples and assessment of PCR inhibitor presence}

BALF samples were initially centrifuged (Centrifuge 5804 $\mathrm{R}$, Eppendorf, Germany) at $13,000 \mathrm{~g}$, at $4{ }^{\circ} \mathrm{C}$ for $20 \mathrm{~min}$. The sample's supernatant was discarded, and the pellet resuspended in $250 \mu \mathrm{L}$ of sterilized DNase and RNase-free PBS (pH: 7.4; Sigma-Aldrich, USA). To extract the DNA from tissue samples, $0.5 \mathrm{~g}$ of lung tissue and $0.2 \mathrm{~g}$ of trachea tissue were used. Regarding DNA extraction from liquid samples, $250 \mu \mathrm{L}$ of resuspended BALF pellet and $500 \mu \mathrm{L}$ of PBS from nasal and laryngeal swabs were used in an in-house protocol previously published [27]. The concentration and purity of the extracted samples were assessed through spectrophotometry (Nanodrop', Thermo Fisher- USA). Overall, tissue samples presented a similar DNA concentration $(900 \mathrm{ng} / \mu \mathrm{L})$, but the ones with different value were either diluted or re-extracted to obtain an equal DNA concentration in each sample. The DNA samples were stored in $200 \mu \mathrm{L}$ microtubes DNAse and RNAse-free (Axygen, USA) and kept at $-20^{\circ} \mathrm{C}$ until use. The presence of PCR inhibitors in the extracted DNA was 
Table 5 Microscopic lesion grading criteria adopted for scoring tracheal samples of Mycoplasma hyopneumoniae (strain 232) experimentally infected pigs

\begin{tabular}{ll}
\hline Score & Lesion description \\
\hline $\mathbf{0}$ & No lesion \\
$\mathbf{1}$ & Epithelium hyperplasia, ciliated cells present in standard quantity with dense cilia \\
$\mathbf{2}$ & Epithelium hyperplasia, reduced cilia density and mild presence of hemorrhage \\
$\mathbf{3}$ & Epithelium hyperplasia with largely reduced cilia density, glandular hyperplasia and increased number of goblet cells \\
$\mathbf{4}$ & Epithelium hyperplasia, glandular hyperplasia, increased number of goblet cells and presence of inflammatory cells infiltration \\
\hline
\end{tabular}

assessed by performing a conventional PCR protocol for the endogenous gene of Glyceraldehyde-3-Phosphate Dehydrogenase (gapdh) gene, using a previously published protocol [28]. To get tested with qPCR, the DNA samples had to be positive in the conventional PCR targeting the gapdh gene.

\section{Detection and absolute quantification of $M$.} hyopneumoniae by qPCR in biological samples

M. hyopneumoniae DNA detection was performed with the aid of qPCR in tracheal and lung samples, nasal swabs, and BALF. All samples were tested in duplicates, using M. hyopneumoniae specific primer pair and hydrolysis probe from a previously published protocol [29].

The reaction was composed of $1 \mathrm{X}$ Master mix Go taq ${ }^{\bullet}$ (Promega, Madison, USA), $0.5 \mu \mathrm{M}$ of each primer (Invitrogen, USA), $0.3 \mu \mathrm{M}$ of the hydrolysis probe (IDT, Iowa City- USA), ultrapure water q.s.p and $1 \mu \mathrm{L}$ of DNA template, adding up to a final reaction volume of $10 \mu \mathrm{L}$. The real-time thermocycler used was a CFX-96 (Biorad USA) model. The cycling conditions were: one cycle of initial denaturation at $95^{\circ} \mathrm{C}$ for $3 \mathrm{~min}$, followed by $39 \mathrm{cy}$ cles of $95^{\circ} \mathrm{C}$ for $15 \mathrm{~s}$, and annealing/extension at $55.7^{\circ} \mathrm{C}$ for $1 \mathrm{~min}$. Quantification results were only used if the $\mathrm{Cq}$ difference was lower than 0.5 cycle [16]. In case of $\mathrm{Cq}$ difference higher than 0.5 cycle, the samples were tested again in triplicates.

Absolute quantification and detection limits were performed using standard curves. The curve consisted of duplicate 10 -fold dilutions, starting at $10^{7}$ copies $\mu \mathrm{L}$ until $10^{1}$ copies $\mu \mathrm{L}$, of a synthetic DNA positive control (GBlock ${ }^{\oplus}$, IDT, Iowa City, IA, USA) of the $150 \mathrm{bp}$ amplified fragment. In addition, quantification data was only used if qPCR efficiency was between 90 and 105\% [30].

\section{Gross lung lesion scoring}

Lungs of both inoculated and control animals were scored (before tissue sample collection) for characteristic lesions of $M$. hyopneumoniae infection, like cranioventral consolidation lesions in the apical, intermediate, accessory lobes and the cranial part of diaphragmatic lobes. Briefly, each of the lobes was visually assessed, and the percentage of the affected area was estimated and further multiplied by the correction factor, based on each lobe's proportional weight in the total lung weight, as described previously [17]. Gross lesions that were not characteristic of $M$. hyopneumoniae infection were not scored in this study.

\section{Microscopic lesion scoring in the tracheal samples}

Tracheal samples were submitted to standard histopathology processing. The slides were then stained with Hematoxylin-Eosin and evaluated using a light microscope. The scoring process of the microscopic lesion was based on the description of the M. hyopneumoniae characteristic microscopic lesions in tracheas [31]. Lesions were scored from 0 to 4 according to the criteria shown in Table 5. A blinded pathologist performed the lesion scoring by checking ten random microscopy fields.

\section{Data analysis}

All numeric data was analyzed using the $\mathrm{R}$ software ( $\mathrm{R}$ Core Team, 2018). Data normality was assessed through the Shapiro-Wilk (SW) test, and homoscedasticity was tested using the Bartlett test, both with 95\% confidence level $(p<0.05)$. Significant differences in parametric data were tested through analysis of variance (ANOVA), and multiple pairwise comparisons were made using the Tukey test and Duncan test $(p<0.05)$. Significant differences in non-parametric data were assessed through Wilcoxon and Kruskal-Wallis tests, and multiple comparisons between data sets were made by using Dunn's test $(p<0.05)$. For non-parametric comparison of repeated measurements, the Friedmann test was used $(p<$ $0.05)$, while for parametric datasets a linear mixedeffects models adjusted for Tukey $(p<0.05)$ were used. Correlations were tested using Pearson's correlation (parametric models) test or Spearman's correlation rank test (non-parametric models).

Authors' contributions

HMSA, LGO and HJM conceived and planned the experiments. HMSA, KS, MLMD, IRHG, and MFM carried out the experiment. HMSA, MLMD, KS, PEBM contributed to sample preparation and data analysis. HMSA, LGO, HJM, DM contributed to the interpretation of the results. HMSA took the lead in writing the manuscript. All authors provided critical feedback and helped shape the research, analysis, and manuscript. The author(s) read and approved the final manuscript. 


\section{Funding}

The authors would like to acknowledge the São Paulo Research Foundation (FAPESP) for funding this study (Grants \#2016 / 18698-2 and \#2019/197104). This study was financed in part by the Coordenação de Aperfeiçoamento de Pessoal de Nível Superior - Brasil (CAPES) - Finance Code 001.

\section{Availability of data and materials}

The datasets used and/or analyzed during the current study are available from the corresponding author on reasonable request.

\section{Declarations}

\section{Ethics approval}

This study was submitted and approved by the São Paulo State University IACUC under the protocol \# 9952 / 16

\section{Competing interests}

The authors declared no conflicts of interest concerning the research authorship, and/or publication of this article.

\section{Author details}

'São Paulo State University (Unesp), School of Agricultural and Veterinarian Sciences, Via de Acesso Prof. Paulo Donato Castellane s/n, Jaboticabal, SP 14884-900, Brazil. 'Faculty of Veterinary Medicine, Ghent University, Ghent, Belgium.

Received: 25 January 2021 Accepted: 3 June 2021

Published online: 30 June 2021

\section{References}

1. Takeuti KL, de Barcellos DESN, de Lara AC, Kunrath CF, Pieters M. Detection of Mycoplasma hyopneumoniae in naturally infected gilts over time. Vet Microbiol. 2017;203(February):215-20. Available from:. https://doi.org/10.10 6/j.vetmic.2017.03.025.

2. Vranckx K, Maes D, Sacristán RDP, Pasmans F, Haesebrouck F. A longitudinal study of the diversity and dynamics of Mycoplasma hyopneumoniae infections in pig herds. Vet Microbiol. 2012;156(3-4):315-21.

3. Sibila M, Pieters M, Molitor T, Maes D, Haesebrouck F, Segalés J. Current perspectives on the diagnosis and epidemiology of Mycoplasma hyopneumoniae infection. Vet J. 2009;181(3):221-31.

4. Maes D, Sibila M, Kuhnert P, Segalés J, Haesebrouck F, Pieters M. Update on Mycoplasma hyopneumoniae infections in pigs: knowledge gaps for improved disease control. Transbound Emerg Dis. 2018;65(3):110-24

5. Thacker EL, Minion C. Diseases of swine. In: Zimmerman JJ, Karriker LA Ramirez A, Schwartz KJ, Stevenson GW, editors. Diseases of swine. 10th ed. Ames: Wiley; 2012. p. 779-97.

6. Otagiri Y, Asai T, Okada M, Uto T, Yazawa S, Hirai H, et al. Detection of Mycoplasma hyopneumoniae in lung and nasal swab samples from pigs by nested PCR and culture methods. J Vet Med Sci. 2005;67(8):801-5.

7. Kwon D, Choi C, Chae C. Chronologic localization of Mycoplasma hyopneumoniae in experimentally infected pigs. Vet Pathol. 2002;39(5):584-7.

8. Sibila M, Nofrarías M, López-Soria S, Segalés J, Riera P, Llopart D, et al. Exploratory field study on Mycoplasma hyopneumoniae infection in suckling pigs. Vet Microbiol. 2007;121(3-4):352-6.

9. Redondo E, Masot AJ, Fernández A, Gázquez A. Histopathological and immunohistochemical findings in the lungs of pigs infected experimentally with Mycoplasma hyopneumoniae. J Comp Pathol. 2009:140(4):260-70.

10. Liu W, Zhou D, Yuan F, Liu Z, Duan Z, Yang K, et al. Surface proteins mhp390 (P68) contributes to cilium adherence and mediates inflammation and apoptosis in Mycoplasma hyopneumoniae. Microb Pathog. 2019; 126(October 2018):92-100. https://doi.org/10.1016/j.micpath.2018.10.035.

11. Paes JA, Virginio VG, Cancela M, Leal FMA, Borges TJ, Jaeger N, et al. Proapoptotic effect of a Mycoplasma hyopneumoniae putative type I signal peptidase on PK(15) swine cells. Vet Microbiol. 2017;201(15):170-6.

12. Sponheim A, Alvarez J, Fano E, Schmaling E, Dee S, Hanson D, et al. Comparison of the sensitivity of laryngeal swabs and deep tracheal catheters for detection of Mycoplasma hyopneumoniae in experimentally and naturally infected pigs early and late after infection. Vet Microbiol. 2019; 241(November 2019):108500. https://doi.org/10.1016/j.vetmic.2019.108500.
13. Vangroenweghe F, Willems E, Malášek J, Thas O, Maes D. Use of tracheabronchial swab qPCR testing to confirm Mycoplasma hyopneumoniae seropositivity in an SPF breeding herd. Porc Health Manag. 2018;4:1-6.

14. Blanchard B, Vena MM, Cavalier A, Le Lannic J, Gouranton J, Kobisch M. Electron microscopic observation of the respiratory tract of SPF piglets inoculated with Mycoplasma hyopneumoniae. Vet Microbiol. 1992;30(4): 329-41.

15. Fablet C, Marois C, Kobisch M, Madec F, Rose N. Estimation of the sensitivity of four sampling methods for Mycoplasma hyopneumoniae detection in live pigs using a Bayesian approach. Vet Microbiol. 2010;143(2-4):238-45. https://doi.org/10.1016/j.vetmic.2009.12.001

16. Bustin SA, Benes V, Garson JA, Hellemans J, Huggett J, Kubista M, et al. The MIQE guidelines: minimum information for publication of quantitative realtime PCR experiments. Clin Chem. 2009;55(4):611-22.

17. Christensen G, Sorensen V, Mousing J. christensen.pdf. In: Straw B, D'Allaire SD, Mengeling W, Taylor DJ, editors. Diseases of swine. 8th ed. Ames: lowa State University Press; 1999. p. 913-40.

18. Garcia-Morante B, Segalés J, Fraile L, Pérez de Rozas A, Maiti H, Coll T, et al. Assessment of Mycoplasma hyopneumoniae-induced pneumonia using different lung lesion scoring systems: a comparative review. J Comp Pathol. 2016;154(2-3):125-34.

19. Jacques M, Blanchard B, Foiry B, Girard C, Kobisch M. In vitro colonization of porcine trachea by Mycoplasma hyopneumoniae; 1992. p. 239-47.

20. DeBey MC, Ross RF. Ciliostasis and loss of cilia induced by Mycoplasma hyopneumoniae in porcine tracheal organ cultures. Infect Immun. 1994; 62(12):5312-8.

21. Paes JA, Leal Zimmer FMA, Moura H, Barr JR, Ferreira HB. Differential responses to stress of two Mycoplasma hyopneumoniae strains. J Proteomics. 2019; 199(March):67-76. https://doi.org/10.1016/j.jprot.2019.03.006.

22. Pieters M, Pijoan C, Fano E, Dee S. An assessment of the duration of Mycoplasma hyopneumoniae infection in an experimentally infected population of pigs. Vet Microbiol. 2009;134(3-4):261-6.

23. Vangroenweghe FACJ, Willems $\mathrm{E}_{1}$ Thas $\mathrm{O}$, Maes DGD. Confirmation of Mycoplasma hyopneumoniae in a breeding herd through tracheobronchial swab sampling and PCR. Vet Rec. 2018;183(10):325.

24. Pieters M, Daniels J, Rovira A. Comparison of sample types and diagnostic methods for in vivo detection of Mycoplasma hyopneumoniae during early stages of infection. Vet Microbiol. 2017;203:103-9. https://doi.org/10.1016/j. vetmic.2017.02.014.

25. Sørensen V, Ahrens P, Barfod K, Feenstra AA, Feld NC, Friis NF, et al. Mycoplasma hyopneumoniae infection in pigs: duration of the disease and evaluation of four diagnostic assays. Vet Microbiol. 1997;54(1):23-34.

26. Bustin SA, Nolan T. Pitfalls of quantitative real- time reverse-transcription polymerase chain reaction. J Biomol Tech. 2004;15(3):155-66.

27. Kuramae-Izioka. A rapid, easy and high yield protocol for total genomic DNA isolation from Colletotrichum gloeosporioides and Fusarium oxysporum for RAPD. Rev Unimar. 1997;19(August):683-9.

28. Birkenheuer AJ, Levy MG, Breitschwerdt EB. Development and evaluation of a seminested PCR for detection and differentiation of Babesia gibsoni (Asian genotype) and B. canis DNA in canine blood samples. J Clin Microbiol. 2003; 41(March):4172-7.

29. Fourour S, Fablet C, Tocqueville V, Dorenlor V, Eono F, Eveno E, et al. A new multiplex real-time TaqMan ${ }^{\oplus}$ PCR for quantification of Mycoplasma hyopneumoniae, M. hyorhinis and M. flocculare: exploratory epidemiological investigations to research mycoplasmal association in enzootic pneumonia-like lesions in slaughtered pigs. J Appl Microbiol. 2018, 125(2):345-55.

30. Kralik $P$, Ricchi M. A basic guide to real time PCR in microbial diagnostics: Definitions, parameters, and everything. Front Microbiol. 2017:8(FEB):1-9.

31. Maxie MG, Jubb KVF, Kennedy PC, Palmer N. Jubb, Kennedy, and Palmer's pathology of domestic animals. Edinburgh: Elsevier Saunders; 2015.

\section{Publisher's Note}

Springer Nature remains neutral with regard to jurisdictional claims in published maps and institutional affiliations. 